\title{
Emerging patient safety issues under health care reform: follow-on biologics and immunogenicity
}

This article was published in the following Dove Press journal:

Therapeutics and Clinical Risk Management

6 December 2011

Number of times this article has been viewed

\author{
Bryan A Liang ${ }^{1-3}$ \\ Timothy Mackey ${ }^{1,4}$ \\ 'Institute of Health Law Studies, \\ California Western School of Law, \\ ${ }^{2}$ Department of Anesthesiology, \\ University of California, San Diego \\ School of Medicine, ${ }^{3}$ San Diego \\ Center for Patient Safety, University \\ of California, San Diego School \\ of Medicine, ${ }^{4}$ Joint Program in Global \\ Health, University of California San \\ Diego-San Diego State University, \\ San Diego, CA, USA
}

\begin{abstract}
US health care reform includes an abbreviated pathway for follow-on biologics, also known as biosimilars, in an effort to speed up access to these complex therapeutics. However, a key patient safety challenge emerges from such an abbreviated pathway: immunogenicity reactions. Yet immunogenicity is notoriously difficult to predict, and even cooperative approaches in licensing between companies have resulted in patient safety concerns, injury, and death. Because approval pathways for follow-on forms do not involve cooperative disclosure of methods and manufacturing processes by innovator companies and follow-on manufacturers, the potential for expanded immunogenicity must be taken into account from a risk management and patient safety perspective. The US Institute of Safe Medication Practices (ISMP) has principles of medication safety that have been applied in the past to high-risk drugs. We propose adapting ISMP principles to follow-on biologic forms and creating systems approaches to warn, rapidly identify, and alert providers regarding this emerging patient safety risk. This type of system can be built upon and provide lessons learned as these new drug forms are developed and marketed more broadly.
\end{abstract}

Keywords: biosimilars, follow-on biologics, immunogenicity, patient safety, law, health care reform

\section{Introduction}

The much-anticipated passage of US health care reform has created opportunities for improving quality and safety in health care. ${ }^{1}$ However, changes in public policy, like changes in the delivery system, have an impact on system function and may create new vulnerabilities despite quality and safety provisions in the law.

One key policy created by health care reform is a new pathway for approval of "follow-on biologics," also known as "biosimilars." This pathway, formally known as the Biologics Price Competition and Innovation Act (BPCIA) within the Patient Protection and Affordable Care Act reform law, would theoretically speed nonbranded forms of biotechnology-derived therapeutic protein products to market at lower prices, akin to the Hatch-Waxman Act that sped small molecule generic drug form development and market entry. ${ }^{2}$

Yet original and copied biologic drugs have had safety challenges, primarily immunogenicity, where the drug induces an unwanted immune reaction in the human body. Immunogenicity is generally unrecognized in clinical care as a safety vulnerability for patients and a drain on health care resources for patients who develop antidrug antibodies and no longer respond to therapy. ${ }^{3}$ However, with the passage of policy that incentivizes follow-on drug forms, which have immunogenicity risks, health care safety leaders 
should expressly note this potential and act to address them in dynamic assessment of local delivery systems.

\section{Immunogenicity: branded forms}

At the outset, therapeutic protein recombinant forms have immunogenicity potential because of their size, living cellbased manufacture, and protein configuration, compared with small molecule, solid-chemical pills. Yet predicting immunogenicity is notoriously difficult. ${ }^{4}$ Immunogenicity appears related to a broad array of divergent factors, including the molecule's structure, patient genetics, type of biologic, impurities, and other factors including the route of administration and frequency of use. ${ }^{5}$

Compounding their difficulty to predict, immunogenicity reactions can be clinically severe and present a major medical emergency, ${ }^{6}$ clearly necessitating patient safety system attention. For example, existing US Food and Drug Administration (FDA)-approved recombinant drug forms can lead to unrecognized immunogenicity and require long-term clinical surveillance to identify accurately. ${ }^{7}$

In addition, short-term data has revealed that immunogenicity of monoclonal antibodies in biologic therapeutics may have negative impacts on treatment response for conditions such as inflammatory bowel disease, types of arthritis, multiple sclerosis, and Crohn's disease. ${ }^{8}$ Long-term studies of immunogenicity of monoclonal antibodies that leads to antidrug antibody response are also associated with treatment discontinuance and higher disease activity with antidrug antibody formation in more than a quarter $(28 \%)$ of patients over a 3 -year period. ${ }^{8}$ Immunogenicity issues in long-term treatment using human recombinant interferon $\beta$ for multiple sclerosis has also shown the potential to negatively impact therapy. ${ }^{9}$

Yet the interferon $\beta$ work also illustrates the challenges of prediction of immunogenicity effects. The frequency of immunogenicity and the magnitude of neutralizing antibody formation may differ among varying interferon $\beta$ products. This further reifies the need for continuous and long-term surveillance of patients utilizing therapeutic products to ensure complications arising from immunogenicity are adequately identified and addressed.

\section{Immunogenicity: cooperative licensing}

Beyond FDA-approved products, immunogenicity is a critical patient safety concern even when companies cooperatively license to produce a biosimilar product. The case of Epogen (erythropoietin) licensing from a US producer to a European Union (EU) manufacturing context is an important example.

Erythropoeitin is a naturally occurring human protein as well as a biologic drug that promotes red blood cell growth. In the late 1990s, Amgen licensed the exclusive rights to produce the drug to Johnson \& Johnson in the EU (Eprex), while Amgen retained US production and sales (Epogen). Both purportedly used the same methodology to produce the drug. ${ }^{5}$ Johnson \& Johnson made several, what it considered minor, manufacturing changes in its production of Eprex. After about 2 years, however, multiple patients taking Eprex in the EU developed a rejection reaction to the drug resulting in pure red cell aplasia, a severe and life-threatening condition where the bone marrow ceases to produce red blood cells. No such effect was observed in patients taking Epogen.

Investigation revealed that Eprex had a different immunogenicity profile than Epogen, with patients developing antibodies to the new formulation. ${ }^{10}$ Although this alone was cause for alarm, beyond creating a rejection reaction to the drug, the new immunogenicity created antibodies against the patients' own naturally occurring erythropoietin. This resulted in the patients' immune systems attacking their own body's erythropoietin, as well as a cross-reactivity response to other medicinal forms of the biologic beyond Eprex. The outcome was that patients could not produce red blood cells using their own or other biologic forms of the drug. Ultimately, severals patient died and others became permanently transfusion-dependent. A combination of high dose immunosuppressive therapy and renal transplantation has been required to treat many of these victims. The results of seemingly minor changes in the biologic had a tremendous negative clinical impact on these patients.

The clinical concerns over this event resulted in intensive investigation. Yet despite continuing research efforts, no clear conclusions have emerged. A wide array of theories has been offered, including different carbohydrate structures, route of administration, a change in the stabilizer, a change in the rubber stopper, and an aggregation of these and other factors. ${ }^{5}$ The debate and search continues for the etiology or etiologies underlying the severe reactions associated with the biologic. ${ }^{11}$

\section{Follow-on biologics: emerging safety concerns}

Though safety concerns regarding this incident have yet to be resolved, recent testing of an Epogen follow-on biologic product by Hospira Inc have begun in the US. ${ }^{12}$ 
Other companies have also announced plans to enter into the follow-on market. Thus, delivery systems may face these policy-created patient safety challenges sooner rather than later.

The interferon $\beta$, Epogen/Eprex, as well as other examples show the sensitive and complex nature of human reactivity to biologics. ${ }^{13}$ Importantly, the research, testing, and technology supporting assessment of biologics were well known to regulatory authorities and industry and full product reviews had been performed. Yet despite this process, severe clinical problems resulted and have yet to be explained sufficiently. Indeed, even the same manufacturing entity may experience immunogenicity difficulties in their products when relatively minor conditions are changed.

Under health care reform, these challenges will likely be magnified. Through empowering the FDA to create an abbreviated approval pathway for biologic products, follow-on biologics will likely become a greater portion of the therapeutic arsenal to treat complex and co-morbid disease. Of particular concern is the lack of specific details regarding analytical, animal, and clinical data needed to support a designation of a biological product as being "biosimilar." Given the presence of immunogenicity in patients both in short-term and long-term studies, careful consideration of the inherent limitations of supporting clinical trial data in detecting immunogenicity risks in patient populations needs to be taken into account. Indeed, there are challenges with assaying all therapeutic proteins, including innovator products. Because of their inherent complexity, simply assaying the protein is not sufficient to show comparability.

Beyond simple comparability, the possibility for "interchangeability" classification under the abbreviated pathway, which permits a biosimilar product to be substituted for the prescribed product, as small molecule generic products are substituted for brand name forms, may also lead to increased utilization of such products and subsequent incidence of immunogenicity. Issues with naming follow-on forms that do not specifically identify the manufacturer creates even greater patient safety risks under interchangeability designations. ${ }^{5}$

Immunogenicity may also become more common because the product "following on" the branded biologic will not be cooperatively licensed, nor will manufacturing methods be disclosed to the follow-on producer as in the Epogen/Eprex case. As such, there may be significant risk that such biologic product may create immunogenicity due to modification in manufacturing processes and other unforeseen changes compared to its original production.

\section{Safety policy}

Building on the experience with recombinant therapeutic products, patient safety advocates must be keenly aware that immunogenicity is a significant clinical safety issue that should be specifically noted and addressed. But further, the public policy favoring these products will create systems issues for health care delivery that require special patient safety consideration. Indeed, the need for attention is urgent: the potential presence of immunogenicity that is not part of the general knowledge or given much attention by safety advocates will be exacerbated by potentially increasing numbers of unexplained immune reactions under health care reform incentives.

To address this patient safety concern, follow-on biologic products should be added as a category to the US Institute of Safe Medication Practices (ISMP) "High-Alert Medications" because these are drugs for which "the consequences of an error are clearly more devastating to patients." ${ }^{14}$ By doing so, the patient safety community will be alerted to the importance of monitoring these drug forms.

Once recognized as a high-alert medication, patient safety advocates and teams should consider using established ISMP tools to design safety systems to avoid preventable error with these medications as well as create systems resilient to its presence. Depending on the local health care delivery infrastructure, system interventions improving access to information about these drugs should first be put into place. Warnings as to immunogenicity posted with the drug in the pharmacy, on physician order entry, and on dispensing records should be prominently displayed and attached, and recurrent in-service training for provider teams should emphasize this adverse event potential. Further, consistent with provisions to engage patients as part of the patient safety team, patients who are taking follow-on biologic drugs should also be provided with layperson, understandable information as part of informed consent, regarding the risks of immunogenicity with follow-on biologics, and areas to assess that may provide early warnings of any immunogenicity reactions. Since the patient and caregivers are the last barrier to harm, it is especially important to engage them as members of the safety team and provide information to empower them to identify potential issues with these therapeutic forms. Of course, this latter should be sensitive to the patient and caregiver competency, ability, and wishes.

In addition, at least on the inpatient level, follow-on biologics access should be limited as other high-alert medications are. Use of a locked cabinet and signout forms with immunogenicity warnings in combination with education 
can highlight the importance of immunogenicity risk to those who will dispense the drug at the pharmacy and on the hospital floors.

The use of auxiliary labels and automated alerts, if the local health IT infrastructure can accommodate it, for follow-on form warnings and patient safety redundancy can also be beneficial as a safety intervention. An auxiliary label that is amenable to immunogenicity warnings transmitted automatically to the physician who ordered the drug can also provide redundancy in clinical information and potential risks associated with these drug forms. Taking a page from drug company direct to consumer advertising and use of the Internet, ${ }^{15}$ using mobile handset technology may be an appropriate system to employ for these warnings.

These efforts should be coupled with implementation of a robust patient surveillance system as a condition of FDA approval of all follow-on biologic products. Such a system could build upon previous reform policy proposals for offlabel promotion of orphan drugs that require manufacturers to submit a pharmacovigilance and risk management plan. ${ }^{16}$ Part of this plan should include provisions for monitoring and regular immunogenicity testing, which would identify potential emerging adverse reactions and can avoid immunogenicity-related ineffective treatment.

In addition, we believe that standardization of purchase and use of these products is essential. Facilities ordering follow-on products should consider purchasing only one brand to provide rapid identification of the product that resulted in immunogenicity, or at the very least, have systems in place to clearly identify the company and version of the follow-on product purchased. Further, protocols in administration of follow-on products and express notation of immunogenicity potential should be put into place, relevant to the specific disease state. As well, clinical practice guidelines, US Preventive Services Task Force recommendations, UK National Institute for Health and Clinical Excellence, and other recognized therapeutic guidance sources should incorporate immune status as a regular assessment in these situations.

These policy approaches may require legislation, which may be difficult to drive forward. This emphasizes the need for cooperative approaches between patients, providers, and safety leaders. Key safety organizations can play a significant role without legislation through voluntary engagement in education and attention to immunogenicity concerns as outlined above. Key drug safety groups such as the Institute for Safe Medication Practices and American Pharmacists Association, patient safety organizations with provider and patient representation including the National Patient Safety Foundation and the WHO World Alliance for Patient Safety, and brand and generic pharmaceutical manufacturer organizations including the Pharmaceutical Research and Manufacturers of America and Generic Pharmaceutical Association, as well as the International Pharmaceutical Federation and the European Generics Association can create an alliance for follow-on biologic safety, and should work jointly in educational endeavors to inform the public regarding the need for vigilance regarding follow-on biologics. Further, an umbrella entity of these organizations can work together with drug regulatory agencies in public-private partnerships to leverage key knowledge from each to adopt, maintain, and update best practices in follow-on biologic safety. Public-private partnerships have become important in addressing important health policy concerns, ${ }^{17}$ and may have significant benefit for drug safety in this setting. A public-private, global health approach for safety of follow-on biologics would also provide important lessons learned and proactive safety planning as these molecules become more integrated into treatment in emerging and developing country environments.

\section{Conclusion}

Addressing dynamic patient safety issues is difficult. In cases of immunogenicity and biologic drugs, limited scientific knowledge as to predictability, rare potential occurrence, but severe negative consequences if an adverse event occurs, create the perfect storm of system gaps that can align to allow significant harm to reach the patient. Furthermore, this potential is magnified by policy created to incentivize the production of follow-on drug forms but fails to adequately address extant patient safety risks. Beyond a focus on local systems and pathways of care, patient safety advocates and policymakers should take into account these challenges associated with new public policies. By doing so, they may be able to proactively design systems to avoid preventable error and be resilient to challenges created by biologics, their follow-on forms, and public policy that incentivizes their production and use.

\section{Disclosure}

The authors report no conflict of interest in this work.

\section{References}

1. Liang BA, Mackey T. Quality and safety in medical care: what does the future hold? Arch Path Lab Med. 2011;135(11):1425-1431.

2. Pub. L. No. 98-4176, 98 Stat. 1585 (1984).

3. Lin RJ. The biological response to biologics. Sci Transl Med. 2011;3(80):80ec61. 
4. Hermeling S, Crommelin DJ, Schellekens H, Jiskoot W. Structureimmunogenicity relationships of therapeutic proteins. Pharm Res. 2004;21(6):897-903.

5. Liang BA. Regulating follow-on biologics. Harv J Legis. 2007;44(2): 363-471.

6. Chamberlain P. Immunogenicity of therapeutic proteins: Part 1: Causes and clinical manifestations of immunogenicity. Regulatory Rev. 2002;5:4-9.

7. Lusher JM, Scharrer I. Evolution of recombinant factor VIII safety: KOGENATE and Kogenate FS/Bayer. Int J Hermatol. 2009;90(4):446-454.

8. Bartelds GM, Krieckaert CL, Nurmohamed MT, et al. Development of antidrug antibodies against adalimumab and association with disease activity and treatment failure during long-term follow-up. JAMA. 2011;305(14):1460-1468.

9. Grossberg SE, Oger J, Grossberg LD, Gehchan A, Klein JP. Frequency and magnitude of interferon $\beta$ neutralizing antibodies in the evaluation of interferon $\beta$ immunogenicity in patients with multiple sclerosis. J Interferon Cytokine Res. 2011. [Epub ahead of print.]

10. Bennett CL, Cournoyer D, Carson KR, et al. Long-term outcome of individuals with pure red cell aplasia and antierythropoietin antibodies in patients treated with recombinant epoetin: a follow-up report from the Research on Adverse Drug Events and Reports (RADAR) Project Blood. 2005;106(10):3343-3347.
11. Schellekens H, Jiskoot W. Eprex-associated pure red cell aplasia and leachates. Nat Biotechnol. 2006;24(6):613-614.

12. Japsen B. Hospira to test generic anemia drug. [Internet]. Chicago Tribune. 2010. Available from: http://articles.chicagotribune.com/201007-28/business/ct-biz-0729-notebook-health-20100728_1_hospirabiosimilar-biotech-drugs. Accessed October 14, 2011.

13. Locatelli F, Roger S. Comparative testing and pharmacovigilance of biosimilars. Nephrol Dial Transplant. 2006;21 Suppl 5:v13-v16.

14. Institute for Safe Medication Practices. ISMP's List of High-Alert Medications. 2008. Available from: http://www.ismp.org/Tools/ highalertmedications.pdf. Accessed October 12, 2011.

15. Liang BA, Mackey T. Direct-to-consumer advertising with interactive internet media: global regulation and public health issues. JAMA. 2011;305(8):824-825.

16. Liang BA, Mackey T. Health care policy. Reforming offlabel promotion to enhance orphan disease treatment. Science. 2010;327(5963):273-274.

17. Buse K, Harmer AM. Seven habits of highly effective global publicprivate health partnerships: practice and potential. Soc Sci Med. 2007;64(2):259-271.
Therapeutics and Clinical Risk Management

\section{Publish your work in this journal}

Therapeutics and Clinical Risk Management is an international, peerreviewed journal of clinical therapeutics and risk management, focusing on concise rapid reporting of clinical studies in all therapeutic areas, outcomes, safety, and programs for the effective, safe, and sustained use of medicines. This journal is indexed on PubMed Central, CAS,

\section{Dovepress}

EMBase, Scopus and the Elsevier Bibliographic databases. The manuscript management system is completely online and includes a very quick and fair peer-review system, which is all easy to use. Visit http://www.dovepress.com/testimonials.php to read real quotes from published authors.

Submit your manuscript here: http://www.dovepress.com/therapeutics-and-clinical-risk-management-journal 